Cite this article as: Chitanda, J.M., Zhang, H., Pahl, E. et al. J. Am. Soc. Mass Spectrom. (2016) 27: 1686.

Final publication is available at Springer via http://dx.doi.org/10.1007/s13361-016-1454-5.

\title{
The Development of Novel Nanodiamond Based MALDI Matrices for the Analysis of Small Organic Pharmaceuticals
}

\author{
Jackson M. Chitanda, Haixia Zhang, Erica Pahl, Randy W. Purves and Anas El-Aneed \\ University of Saskatchewan, Saskatoon, Canada
}

\begin{abstract}
The utility of novel functionalized nanodiamonds (NDs) as matrices for matrix-assisted laser desorption ionization-mass spectrometry (MALDI-MS) is described herein. MALDI-MS analysis of small organic compounds ( $<1000 \mathrm{Da}$ ) is typically complex due to interferences from numerous cluster ions formed when using conventional matrices. To expand the use of MALDI for the analysis of small molecules, , novel matrices were designed by covalently linking conventional matrices (or a lysine moiety) to detonated NDs. Four new functionalized NDs were evaluated for their ionization capabilities using five pharmaceuticals with varying molecular structures. Two ND matrices were able to ionize all tested pharmaceuticals in the negative ion mode, producing the deprotonated ions $[\mathrm{M}-\mathrm{H}]^{-}$. Ion intensity for target analytes was generally strong with enhanced signal-to-noise ratios compared with conventional matrices. The negative ion mode is of great importance for biological samples as interference from endogenous compounds is inherently minimized in the negative ion mode. Since the molecular structures of the tested pharmaceuticals did not suggest that negative ion mode would be preferable, this result magnifies the importance of these findings. On the other hand, conventional matrices primarily facilitated the ionization as expected in the positive ion mode, producing either the protonated molecules $[\mathrm{M}+\mathrm{H}]^{+}$or cationic adducts (typically producing complex spectra with numerous adduct peaks). The data presented in this study suggests that these matrices may offer advantages for the analysis of low molecular weight pharmaceuticals/metabolites.
\end{abstract}

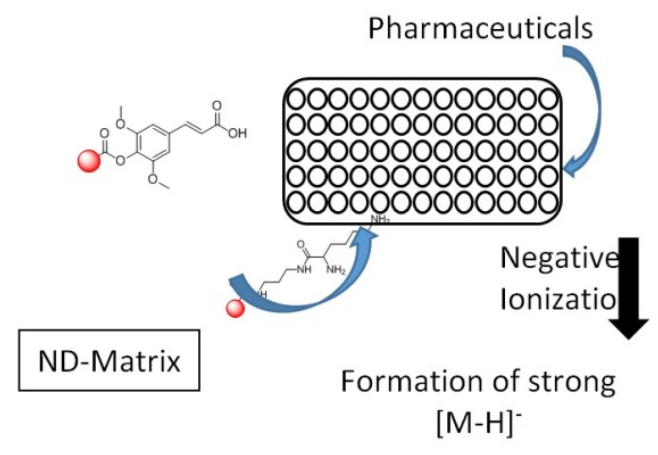


Cite this article as: Chitanda, J.M., Zhang, H., Pahl, E. et al. J. Am. Soc. Mass Spectrom. (2016) 27: 1686. Final publication is available at Springer via http://dx.doi.org/10.1007/s13361-016-1454-5.

\section{Introduction}

Matrix-Assisted Laser Desorption Ionization (MALDI) is a soft ionization technique that allows for the analysis of a wide array of organic compounds and biomaterials by mass spectrometry (MS). MALDI has been used for both qualitative and quantitative applications,[1, 2] and is widely utilized for MS-based imaging.[3-5] MALDI-MS requires the use of a matrix that is premixed with the analyte prior to analysis. The majority of matrices are small organic compounds that will interfere, to a large extent, with the analysis of small molecules $(\mathrm{MW}<1,000)$, such as pharmaceuticals, because they form several intense cluster ions in the low $\mathrm{m} / \mathrm{z}$ range. Because such clusters decrease spectral quality thereby increasing detection limits, the analysis of small molecules such as pharmaceuticals is primarily carried out by other techniques such as electrospray ionization (ESI). However, MALDI offers several advantages over ESI including a simplified sample preparation, the formation of predominantly singly charged ions (which simplifies mass spectra), tolerance for additives and salts, and a much faster speed of analysis.[6, 7]

To expand the use of MALDI-MS to small organic compounds, various strategies have been employed such as utilizing nanoparticle matrices with high molecular weights[8, 9], or immobilizing conventional matrices on nanoparticle or polymeric surfaces.[10,11] In fact, both metal- and carbon-based nanoparticles have been evaluated.[12-15] For example, gold nanoparticles were recently shown to enhance the analysis of small organic compounds with diverse polarity, such as amino acids, saccharides, nucleosides, and nucleic bases.[16] However, enrichment of sodium was intentionally applied so that sodiated adducts are formed to facilitate the MALDI-MS analysis.[16] Gold nanoparticles were also surface-modified with $\alpha$-Cyano-4hydroxycinnamic acid (CHCA) conventional matrix for the effective analysis of protein digests.[17] Another class of carbon-based nanoparticles are nanodiamonds (ND) with their main use in MALDI analysis being linked to sample preparation and clean-up.[18-21] Particularly, they are used to enhance the isolation of biomaterials, such as carbohydrates and proteins, prior to MALDI-MS analysis[21-24]; in such applications, nanodiamonds are utilized in conjunction with conventional matrices.

In this work, we describe the development of novel nanodiamond-based matrices via the functionalization of the ND surface. Linking moieties to a diamond surface for biotechnology applications was reported in the past, such as the early work by Ushizawa et. al. in which DNA was covalently linked to oxidized diamond surfaces.[25] In a similar strategy, we covalently linked conventional matrices and active moieties to the surface of detonated ND. The main idea is to take advantage of the optical properties of the nanodiamonds[26, 27] and to combine this with the ionization ability of conventional matrices for the analysis of small organic compounds. These novel matrices were evaluated for the analysis of pharmaceuticals showing signal enhancement in comparison to conventional matrices.

\section{Experimental Section}

Materials: Diamond nanoparticles, of diameter 4-5 nm and zeta potential - $45 \mathrm{~V}$, were obtained from International Technology Centre (Raleigh, NC, USA). The nanodiamonds had carboxylic acid and hydroxyl ion functionalities on the surface. $\mathrm{Sp}^{3}$ content (diamond content) was 75-99 $\mathrm{wt} \%$. Nanodiamonds were subjected to reoxidation using a mixture of concentrated $\mathrm{H}_{2} \mathrm{SO}_{4}$ and

University of Saskatchewan Author Manuscript, available October 1, 2017. 
Cite this article as: Chitanda, J.M., Zhang, H., Pahl, E. et al. J. Am. Soc. Mass Spectrom. (2016) 27: 1686. Final publication is available at Springer via http://dx.doi.org/10.1007/s13361-016-1454-5.

$\mathrm{HNO}_{3}(3: 1 \mathrm{v} / \mathrm{v})$ under bath sonication as described.[28] This process converted most of the surface functionalities to carboxylate groups thus homogenizing the surface for subsequent reactions.

a-cyano-4-hydroxycinnamic acid (CHCA), 2,5-dihydroxybenzoic acid (DHB), sinapinic acid (SA) and thionyl chloride, used for the synthesis of functionalized ND, were sourced from Sigma Aldrich (St. Louis, MO). Piroxicam (PX), tetracycline (TC), erythromycin (ER), quinine (QN), prednisolone (PD) powders were obtained from Extrasynthese (Genay, France).

CHCA and SA powder used as controls during MALDI were obtained from Waters (Milford, MA). DHB was obtained from Ettan Chemicals (Uppsala, Sweden). All organic solvents (methanol (MeOH), acetonitrile (ACN) and dimethyl formamide (DMF)) were of analytical grade and were mass spectrometry-compatible.

YTZ ${ }^{\circledR}$ (Yttrium Stabilized Zirconia) grinding media $(0.05 \mathrm{~mm})$ were obtained from Tosoh Corporation (Grove City, OH). These zirconia Beads are high density, highly spherical products used to disaggregate nanodiamonds from their core aggregates. They are made of a metallic blend of zirconium oxide and yttrium oxide $\left(95 \% \mathrm{ZrO}_{2}, 5 \% \mathrm{Y}_{2} \mathrm{O}_{3}\right)$, which when subjected to a degree of ultrasonication hits the graphitic soot and on the diamond core. These collisions induce high energy impact and sheer forces, ultimately causing the aggregation to break. In this process bath sonication additionally creates shock waves adding to the effect of the zirconia beads to produce high intensity impact.

\section{Synthesis of nanodiamond-based matrices}

Formation of ND-COCI: In a dry Schlenk flask, carboxylated nanodiamonds (0.100 g), Zirconium beads $(0.150 \mathrm{~g})$ and DMF $(10 \mathrm{~mL})$ were added and sonicated. After $12 \mathrm{~h}, \mathrm{DMF}$ was evaporated to dryness using a vacuum pump. To the dried mixture, under nitrogen, excess thionyl chloride $(6.0 \mathrm{~mL})$ was added and sonicated for $24 \mathrm{~h}$. Thereafter, the flask was placed on a $100^{\circ} \mathrm{C}$ pre-heated oil bath. After refluxing the reaction for $24 \mathrm{~h}$, it was left to cool to room temperature and then sonicated for $5 \mathrm{~h}$ before placing it back on the oil bath for an additional 24 $\mathrm{h}$ of refluxing. After cooling the reaction mixture to ambient temperature, excess thionyl chloride $\left(\mathrm{SOCl}_{2}\right)$ was removed under high vacuum.

Formation of ND-COO-R: To the dried ND-COCl/Zirconium beads mixture, DMF (15 mL) and the respective organic matrix (CHCA, DHB or SA) were added in large excess (500 mg). The heterogeneous mixture was sonicated for $2 \mathrm{~h}$ then refluxed for $12 \mathrm{~h}$ at $100^{\circ} \mathrm{C}$. Upon cooling, Zirconium beads were separated by pipetting off the DMF solution mixture. The DMF solution mixture was centrifuged at $4^{\circ} \mathrm{C}$ for $25 \mathrm{~min}(\mathrm{RCF}=20000)$. The supernatant was removed and DMF was added to the residue and sonicated again. This procedure was repeated three times in order to ensure a total removal of unreacted conventional matrices. In the last centrifugation cycle, acetone was used instead of DMF. Finally, to the residue, water was added and sonicated for $2 \mathrm{~h}$ before it was lyophilized for $48 \mathrm{~h}$. The final grayish powder was collected and analyzed by thermal gravimetric analysis (TGA). TGA profile, that occurred at varying temperatures, showed the mass lost from the surface of the ND, to be larger than the one obtained from pristine ND. A summary of the chemical reaction is shown in Figure 1. The synthesis of ND-Lys followed an already published method in which the ND-Lys was used as a gene delivery system.[28] 
Cite this article as: Chitanda, J.M., Zhang, H., Pahl, E. et al. J. Am. Soc. Mass Spectrom. (2016) 27: 1686. Final publication is available at Springer via http://dx.doi.org/10.1007/s13361-016-1454-5.

The synthesized particles were characterized using Fourier Transform Infrared-Attenuated Total Reflection (Vertex 70 FT-IR-ATR, Bruker Optics Inc., Billerica, MA, USA) and Thermal Gravimetric Analysis (TGA-Q5000, TA Instruments, New Castle, DE, USA). Differential thermal gravimetric (DTG) curves were plotted from the data extracted from the TGA. TGA experiments were conducted using 5-7 mg sample mass and at a ramping rate of $10{ }^{\circ} \mathrm{C} / \mathrm{min}$ from c.a. $30^{\circ} \mathrm{C}$ to $900{ }^{\circ} \mathrm{C}$. Nitrogen was used both as a balance gas and sample gas at a flowrate of 40 and $60 \mathrm{~mL} / \mathrm{min}$, respectively. Weight loss up to $200{ }^{\circ} \mathrm{C}$ was excluded for it is attributed to the loss of moisture and other volatile substances. Surface loading was 1.0, 2.1, and $2.3 \mathrm{mmol} / \mathrm{g}$ for ND-DHB, ND-SA, and ND-CHCA, respectively. For ND-Lys the loading is 1.97[28]

\section{MALDI Analysis}

An AB Sciex 4800 MALDI TOF/TOFTM instrument (Applied Biosystems, Frederick, MD, USA) was operated in MS reflectron mode in both positive and negative ion modes. Laser energy with ranges of 5000-5300 (i.e. 12.7-13.4 $\mu \mathrm{J}$ ) was required for the functionalized ND matrices. The same laser energy range was used for the SA, CHCA and DHB matrices as well as the regular laser power usually used for these matrices. The use of high laser energy with conventional matrices produced no or weak signals in most cases (data not shown). 400 Laser shots were acquired per spot. Calibration of the instrument was achieved for each ND-based matrices by using the signals from their corresponding conventional matrices (monomers, dimers and sodium adducts).

For MALDI-MS analysis, a $1 \mathrm{mg} / \mathrm{mL}$ suspension of the desired ND matrix was formed using a $\mathrm{MeOH}: \mathrm{H}_{2} \mathrm{O}$ :ACN (1:1:1) solvent mixture. The suspension was vortexed and sonicated for 30 min. Respective solutions of $20 \mu \mathrm{g} / \mathrm{mL}$ of the desired analyte were prepared in a MeOH:ACN(1:1) solvent system. $50 \mu \mathrm{L}$ solutions of SA (MeOH:ACN (1:1)), CHCA (MeOH:ACN (1:1)) and DHB $\left(\mathrm{H}_{2} \mathrm{O}: \mathrm{ACN}(1: 1)\right)$ were prepared to give final matrix concentrations of $10 \mathrm{~g} / \mathrm{L}$. ND matrices were vortexed before spotting $1 \mu \mathrm{L}$ of the suspension onto the stainless-steel MALDI plate. The ND matrix was dried at ambient conditions followed by spotting $1 \mu \mathrm{L}$ of the analyte solution on top of the matrix which was dried at room temperature. A similar procedure was followed for SA, CHCA and DHB matrices as control samples. Note that other spotting strategies were evaluated; however, the above method proved to be the best approach.

\section{Results and Discussions}

New matrices were characterized using FT-IR and TGA analysis indicating the production of the target nanodiamond-modified matrices. The production and characterization of ND-Lys was described in detail previously[28] and the following section will focus on ND-SA, ND-CHCA and ND-DHB. Figure 2 clearly shows that the peaks from the synthesized particles are sharper than those of the pristine nanodiamonds and there is a shift in the wavenumber at which the peaks appear. ND-COOH particles showed a broad peak at $3400 \mathrm{~cm}-1$, which is attributed to the hydrogen-bonded vibrations of the hydroxyl $(\mathrm{O}-\mathrm{H})$ groups. The same peaks on the synthesized particles are sharper and appear at higher wavenumbers, ranging from 3628 to $3727 \mathrm{~cm}^{-1}$. The peaks appearing around $2980 \mathrm{~cm}^{-1}$ represent the stretching vibrations of sp2 and sp3 C-H of carboxylic acid hydroxyl (acid $\mathrm{O}-\mathrm{H}$ ) groups. These peaks are seemingly absent from the spectra for the pristine nanodiamonds (ND-COOH). Although the pristine nanodiamonds possess the acid $\mathrm{O}-\mathrm{H}$ groups, the signal may have been buried under the broad peak. The next distinct peak 
Cite this article as: Chitanda, J.M., Zhang, H., Pahl, E. et al. J. Am. Soc. Mass Spectrom. (2016) 27: 1686. Final publication is available at Springer via http://dx.doi.org/10.1007/s13361-016-1454-5.

is the carbonyl group $(\mathrm{C}=\mathrm{O})$ from carboxylic acid and ester functionalities, which appear around 1798 and $1640 \mathrm{~cm}^{-1}$, respectively. In the pristine nanodiamonds, these peaks are weak but become sharper with further functionalization with conventional matrices. While there was a shift to a lower wavenumber for the carboxylic acid $\mathrm{C}=\mathrm{O}$ group (from 1798 to $1172 \mathrm{~cm}^{-1}$ ), there was a higher shift in the ester $\mathrm{C}=\mathrm{O}\left(1628\right.$ to $\left.1658 \mathrm{~cm}^{-1}\right)$. This shift signifies different connectivity of functional groups on the surface of nanodiamonds.

The TGA and DTG profiles of the four particles are shown in Figure 3. The weight loss calculated between 250 and $800{ }^{\circ} \mathrm{C}$ showed that ND-SA had the most weight loss (54.4\%) followed by ND-CHCA (49.0\%). The lowest weight loss was recorded by ND-DHB (18.9\%). Particles' surface loading was determined based on the molar mass of moieties on the surface. The larger the molar mass the smaller coverage will be observed, hence ND-COOH gave the highest surface loading of $5.8 \mathrm{mmol} / \mathrm{g}$ followed by ND-CHCA ( $2.3 \mathrm{mmol} / \mathrm{g})$ and ND-SA (2.1 $\mathrm{mmol} / \mathrm{g})$. ND-DHB $(1.0 \mathrm{mmol} / \mathrm{g})$ gave the least surface loading. This could be attributed to the increased stability of the surface moieties. It is possible that the $1.0 \mathrm{mmol} / \mathrm{g}$ surface loading of ND-DHB is more thermodynamically stable than the higher loading capacity observed for the other modified nanodiamonds which was at least double in value. More characterization at the physicochemical level as well as computational modeling may explain such observation, which is beyond the scope of this article.

The use of functionalized ND as MALDI matrices was evaluated both in the positive and negative ion modes. Surprisingly, two functionalized NDs, namely ND-SA and ND-Lys, produced desirable negative ion mode mass spectra. In particular, strong deprotonated molecules $[\mathrm{M}-\mathrm{H}]^{-}$were produced in comparison to conventional matrices. Tables 1 and 2 summarise the data obtained from functionalized NDs and conventional matrices, respectively. Signal intensity was defined based on observed ion counts with "very intense" being more than $10^{5}$ ion counts, whereas very weak was below $10^{2}$. Background noise was generally below 30 ion counts unless very high energy (i.e., $13.4 \mu \mathrm{J}$ ) was used, which resulted in an ion count of $\sim 150$. Despite the varying and unrelated structures of the target analytes, the use of functionalized NDs in the negative ion mode produced mass spectra with target compounds having reduced signal to noise ratios in comparison with conventional matrices. While four analytes appeared as $[\mathrm{M}-\mathrm{H}]^{-}$in the negative ion mode, quinine ionized as the species $\left[\mathrm{M}-\mathrm{H}_{2}-\mathrm{H}\right]^{-}$, probably due to the formation of a stable conjugated ion. Negative ion mode is of particular interest when using biological samples as interferences from endogenous compounds (i.e., matrix effects) are inherently minimized in comparison with the positive ion-mode.[29] Analyte peaks were absent in controls where only the matrix was spotted onto the MALDI plate, ensuring that the analyte of interest produced the observed ions. Figure 4 illustrates the MS spectra obtained for erythromycin in which the formation of [M-H]- at $\mathrm{m} / \mathrm{z} 732$ was observed when using nanodiamond-modified matrices. As it can be seen, abundant deprotonated ions were observed with no interference from the matrix, whereas this species was not observed in the case of negative ionization using conventional matrices (Figure 4).

The main goal of this work is to develop novel matrices that can ionize various structures of small organic pharmaceuticals. In fact, the current use of conventional matrices for small pharmaceuticals is difficult and typically requires the trial of many matrices to ensure minimum interferences with the analyte of interest. Our group recently analyzed, qualitatively and quantitatively, a gene delivery agent, namely a gemini surfactant, using MALDI-ToF/ToF with

University of Saskatchewan Author Manuscript, available October 1, 2017. 
Cite this article as: Chitanda, J.M., Zhang, H., Pahl, E. et al. J. Am. Soc. Mass Spectrom. (2016) 27: 1686. Final publication is available at Springer via http://dx.doi.org/10.1007/s13361-016-1454-5.

sinapinic acid being the matrix of choice.[30] However, seven different conventional matrices were initially investigated to ensure no matrix interference was observed during the analysis. Such efforts are time-consuming and there is no guarantee a suitable matrix will be found. Our data showed that all tested compounds were strongly ionized in the negative ion mode without interference suggesting that these promising new matrices could be used routinely for the analysis of small pharmaceuticals.

All tested pharmaceuticals contain phenolic, alcohol, tertiary amine, amide or imine functional groups (analyte structures are shown in Tables 1 and 2). Based on the molecular structures of the evaluated pharmaceuticals, the positive ion mode is expected to be favoured during MALDI-MS analysis. In fact, the conventional matrices produced the expected results as shown in Table 2 and Figure 5. Three analytes, namely piroxicam, tetracycline, and erythromycin produced no signal in the negative ion mode while quinine and prednisolone were ionized in the negative ion mode with two of the conventional matrices (Table 2). However, in the positive ion mode, with the exception of quinine, either the expected protonated ions $[\mathrm{M}+\mathrm{H}]^{+}$or sodium or potassium adduct ions were produced. While the functionalized NDs did not facilitate the formation of $[\mathrm{M}+\mathrm{H}]^{+}$in the positive ion mode, adduct formation was observed in the case of NDs (Table 1, Figure 5). Illustrative mass spectra are shown in Figure 5 demonstrating the favorable formation of sodium or potassium adducts for prednisolone at $m / z 383$ and 399, respectively when using nanodiamond-based matrices. Adduct formation was also observed when using conventional matrices, however for SA or DHB matrices the protonated species at $\mathrm{m} / \mathrm{z} 361$ were dominant.

Based on the observed data, two functionalized NDs seem to function as promising MALDI matrices, namely ND-SA and ND-Lys (Table 1). The ability of ND-Lys to effectively serve as MALDI matrix in the negative ion mode can be rationalized by the presence of primary amine functional groups that can capture protons from analytes, producing the desired $[\mathrm{M}-\mathrm{H}]^{-}$species. On the other hand, it is difficult to rationalize the performance of ND-SA based on its structural features. This is not surprising as the exact mechanism of ionization during MALDI-MS is not fully understood,[6] with the vast majority of new matrices being introduced based on experimental data.[31-34] It is this fact that prompted us initially to evaluate a novel drug delivery agent ND-Lys as a MALDI matrix.

\section{Conclusion}

Novel functionalized nanodiamonds produced promising results as new MALDI matrices that can ionize various structures of small organic pharmaceuticals in the negative ion mode as [M$\mathrm{H}]^{-}$. The molecular structures of the tested analytes were variable with molecular weight ranging from 324-733 Da, indicating the applicability of the new matrices on varying structures. The strong formation of [M-H]', particularly when using ND-SA or ND-LYS, is promising and rather unexpected. Interestingly, corresponding conventional matrices failed in producing the desired species during negative ionization. Therefore, the developed new nanodiamond-based matrices may provide an alternative option for analyzing various analyte structures in the negative ion mode where interferences from endogenous compounds within biological samples is inherently minimized. The preliminary results discussed in this study warrant additional investigations; therefore, we will be evaluating these novel matrices with additional pharmaceutical structures. We will also continue to optimize the nanoparticle size, concentration and the preparation method. Future work will involve the evaluation of functionalized NDs matrices with biological extracts, for quantitative analysis and for MALDI imaging. 
Cite this article as: Chitanda, J.M., Zhang, H., Pahl, E. et al. J. Am. Soc. Mass Spectrom. (2016) 27: 1686.

Final publication is available at Springer via http://dx.doi.org/10.1007/s13361-016-1454-5.

\section{References}

1. Ivanova, B., Spiteller, M.: A novel UV-MALDI-MS analytical approach for determination of halogenated phenyl-containing pesticides. Ecotoxicology and environmental safety. 91, 86-95 (2013)

2. Porta, T., Grivet, C., Knochenmuss, R., Varesio, E., Hopfgartner, G.: Alternative CHCAbased matrices for the analysis of low molecular weight compounds by UV-MALDItandem mass spectrometry. Journal of mass spectrometry : JMS. 46, 144-152 (2011)

3. Shanta, S.R., Kim, T.Y., Hong, J.H., Lee, J.H., Shin, C.Y., Kim, K.H., et al.: A new combination MALDI matrix for small molecule analysis: application to imaging mass spectrometry for drugs and metabolites. The Analyst. 137, 5757-5762 (2012)

4. Wang, J., Qiu, S., Chen, S., Xiong, C., Liu, H., Zhang, N., et al.: MALDI-TOF MS imaging of metabolites with a N-(1-naphthyl) ethylenediamine dihydrochloride matrix and its application to colorectal cancer liver metastasis. Analytical chemistry. 87, 422430 (2015)

5. Schone, C., Hofler, H., Walch, A.: MALDI imaging mass spectrometry in cancer research: combining proteomic profiling and histological evaluation. Clinical biochemistry. 46, 539-545 (2013)

6. Awad, H., Khamis, M.M., El Aneed, A.: Mass Spectrometry, Review of the Basics: Ionization. Appl Spectrosc Rev. 50, 185-175 (2015)

7. Cohen, L.H., Gusev, A.I.: Small molecule analysis by MALDI mass spectrometry. Analytical and bioanalytical chemistry. 373, 571-586 (2002)

8. Celikbicak, O., Demirel, G., Piskin, E., Salih, B.: Small molecule analysis using laser desorption/ionization mass spectrometry on nano-coated silicon with self-assembled monolayers. Analytica chimica acta. 729, 54-61 (2012)

9. Taira, S., Sugiura, Y., Moritake, S., Shimma, S., Ichiyanagi, Y., Setou, M.: Nanoparticleassisted laser desorption/ionization based mass imaging with cellular resolution. Analytical chemistry. 80, 4761-4766 (2008)

10. Tseng, M.C., Obena, R., Lu, Y.W., Lin, P.C., Lin, P.Y., Yen, Y.S., et al.: Dihydrobenzoic Acid Modified Nanoparticle as a MALDI-TOF MS Matrix for Soft Ionization and Structure Determination of Small Molecules with Diverse Structures. J Am Soc Mass Spectr. 21, 1930-1939 (2010)

11. Lin, Y.S., Chen, Y.C.: Laser desorption/ionization time-of-flight mass spectrometry on sol-gel-derived 2,5-dihydroxybenzoic acid film. Anal Chem. 74, 5793-5798 (2002)

12. Gholipour, Y., Giudicessi, S.L., Nonami, H., Erra-Balsells, R.: Diamond, titanium dioxide, titanium silicon oxide, and barium strontium titanium oxide nanoparticles as matrixes for direct matrix-assisted laser desorption/ionization mass spectrometry analysis of carbohydrates in plant tissues. Analytical chemistry. 82, 5518-5526 (2010) 
Cite this article as: Chitanda, J.M., Zhang, H., Pahl, E. et al. J. Am. Soc. Mass Spectrom. (2016) 27: 1686. Final publication is available at Springer via http://dx.doi.org/10.1007/s13361-016-1454-5.

13. Ren, S.F., Guo, Y.L.: Carbon nanotubes (2,5-dihydroxybenzoyl hydrazine) derivative as $\mathrm{pH}$ adjustable enriching reagent and matrix for MALDI analysis of trace peptides. Journal of the American Society for Mass Spectrometry. 17, 1023-1027 (2006)

14. Lee, K.H., Chiang, C.K., Lin, Z.H., Chang, H.T.: Determining enediol compounds in tea using surface-assisted laser desorption/ionization mass spectrometry with titanium dioxide nanoparticle matrices. Rapid communications in mass spectrometry : RCM. 21, 2023-2030 (2007)

15. Kong, X., Huang, Y.: Applications of graphene in mass spectrometry. Journal of nanoscience and nanotechnology. 14, 4719-4732 (2014)

16. Sekuła, J., Nizioł, J., Rode, W., Ruman, T.: Gold nanoparticle-enhanced target (AuNPET) as universal solution for laser desorption/ionization mass spectrometry analysis and imaging of low molecular weight compounds Analytica chimica acta. In prerss, (2015)

17. Duan, J.C., Linman, M.J., Chen, C.Y., Cheng, Q.J.: CHCA-Modified Au Nanoparticles for Laser Desorption Ionization Mass Spectrometric Analysis of Peptides. J Am Soc Mass Spectr. 20, 1530-1539 (2009)

18. Kailasa, S.K., Wu, H.F.: Nanomaterial-based miniaturized extraction and preconcentration techniques coupled to matrix-assisted laser desorption/ionization mass spectrometry for assaying biomolecules. Trac-Trend Anal Chem. 65, 54-72 (2015)

19. Kong, X.L., Huang, L.C.L., Hsu, C.M., Chen, W.H., Han, C.C., Chang, H.C.: Highaffinity capture of proteins by diamond nanoparticles for mass spectrometric analysis. Analytical chemistry. 77, 259-265 (2005)

20. Kong, X.L., Huang, L.C.L., Liau, S.C.V., Han, C.C., Chang, H.C.: Polylysine-coated diamond nanocrystals for MALDI-TOF mass analysis of DNA oligonucleotides. Analytical chemistry. 77, 4273-4277 (2005)

21. Wu, C.L., Wang, C.C., Lai, Y.H., Lee, H., Lin, J.D., Lee, Y.T., et al.: Selective Enhancement of Carbohydrate Ion Abundances by Diamond Nanoparticles for Mass Spectrometric Analysis. Analytical chemistry. 85, 3836-3841 (2013)

22. Pham, M.D., Yu, S.S.F., Han, C.C., Chan, S.I.: Improved Mass Spectrometric Analysis of Membrane Proteins Based on Rapid and Versatile Sample Preparation on Nanodiamond Particles. Analytical chemistry. 85, 6748-6755 (2013)

23. Soo, P.C., Horng, Y.T., Chen, A.T., Yang, S.C., Chang, K.C., Lee, J.J., et al.: Validation of nanodiamond-extracted CFP-10 antigen as a biomarker in clinical isolates of Mycobacterium tuberculosis complex in broth culture media. Tuberculosis. 95, 620-624 (2015)

24. Wang, J., Liu, Q., Liang, Y., Jiang, G.B.: Recent progress in application of carbon nanomaterials in laser desorption/ionization mass spectrometry. Anal Bioanal Chem. 408, 2861-2873 (2016) 
Cite this article as: Chitanda, J.M., Zhang, H., Pahl, E. et al. J. Am. Soc. Mass Spectrom. (2016) 27: 1686.

Final publication is available at Springer via http://dx.doi.org/10.1007/s13361-016-1454-5.

25. Ushizawa, K., Sato, Y., Mitsumori, T., Machinami, T., Ueda, T., Ando, T.: Covalent immobilization of DNA on diamond and its verification by diffuse reflectance infrared spectroscopy. Chem Phys Lett. 351, 105-108 (2002)

26. Kratochvilova, I., Kovalenko, A., Fendrych, F., Petrakova, V., Zalis, S., Nesladek, M.: Tuning of nanodiamond particles' optical properties by structural defects and surface modifications: DFT modelling. J Mater Chem. 21, 18248-18255 (2011)

27. Tisler, J., Balasubramanian, G., Naydenov, B., Kolesov, R., Grotz, B., Reuter, R., et al.: Fluorescence and Spin Properties of Defects in Single Digit Nanodiamonds. Acs Nano. 3, 1959-1965 (2009)

28. Kaur, R., Chitanda, J.M., Michel, D., Maley, J., Borondics, F., Yang, P., et al.: Lysinefunctionalized nanodiamonds: synthesis, physiochemical characterization, and nucleic acid binding studies. Int J Nanomedicine. 7, 3851-3866 (2012)

29. Gosetti, F., Mazzucco, E., Zampieri, D., Gennaro, M.C.: Signal suppression/enhancement in high-performance liquid chromatography tandem mass spectrometry. Journal of chromatography. A. 1217, 3929-3937 (2010)

30. Buse, J., Purves, R.W., Verrall, R.E., Badea, I., Zhang, H.X., Mulligan, C.C., et al.: The development and assessment of high-throughput mass spectrometry-based methods for the quantification of a nanoparticle drug delivery agent in cellular lysate. J Mass Spectrom. 49, 1171-1180 (2014)

31. Francese, S., Bradshaw, R., Flinders, B., Mitchell, C., Bleay, S., Cicero, L., et al.: Curcumin: A Multipurpose Matrix for MALDI Mass Spectrometry Imaging Applications. Analytical chemistry. 85, 5240-5248 (2013)

32. Kuang, M., Zhang, Y., Yang, P.Y., Lu, H.J.: Novel Ionic Matrices for Enhanced Ionization of Oligosaccharides/Glycopeptides during MALDI-MS Analysis. Acta Chim Sinica. 71, 1007-1010 (2013)

33. Schinkovitz, A., Kenfack, G.T., Seraphin, D., Levillain, E., Dias, M., Richomme, P.: Selective detection of alkaloids in MALDI-TOF: the introduction of a novel matrix molecule. Analytical and bioanalytical chemistry. 403, 1697-1705 (2012)

34. Shroff, R., Svatos, A.: Proton Sponge: A Novel and Versatile MALDI Matrix for the Analysis of Metabolites Using Mass Spectrometry. Analytical chemistry. 81, 7954-7959 (2009) 


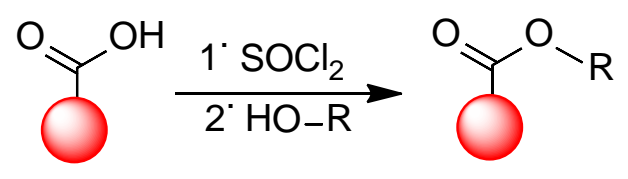<smiles>COc1cc(/C=C/C(=O)O)cc(OC)c1OCc1ccc(/C=C(\C#N)C(=O)O)cc1</smiles>

Figure 1: The chemical reaction leading to the production of ND-COCl, which was then reacted with desired matrices producing ND-SA, ND-CHCA and ND-DHB. The synthesis of ND-Lys followed an already reported method.[28] The ball represents the nanodiamond core 
Cite this article as: Chitanda, J.M., Zhang, H., Pahl, E. et al. J. Am. Soc. Mass Spectrom. (2016) 27: 1686.

Final publication is available at Springer via http://dx.doi.org/10.1007/s13361-016-1454-5.

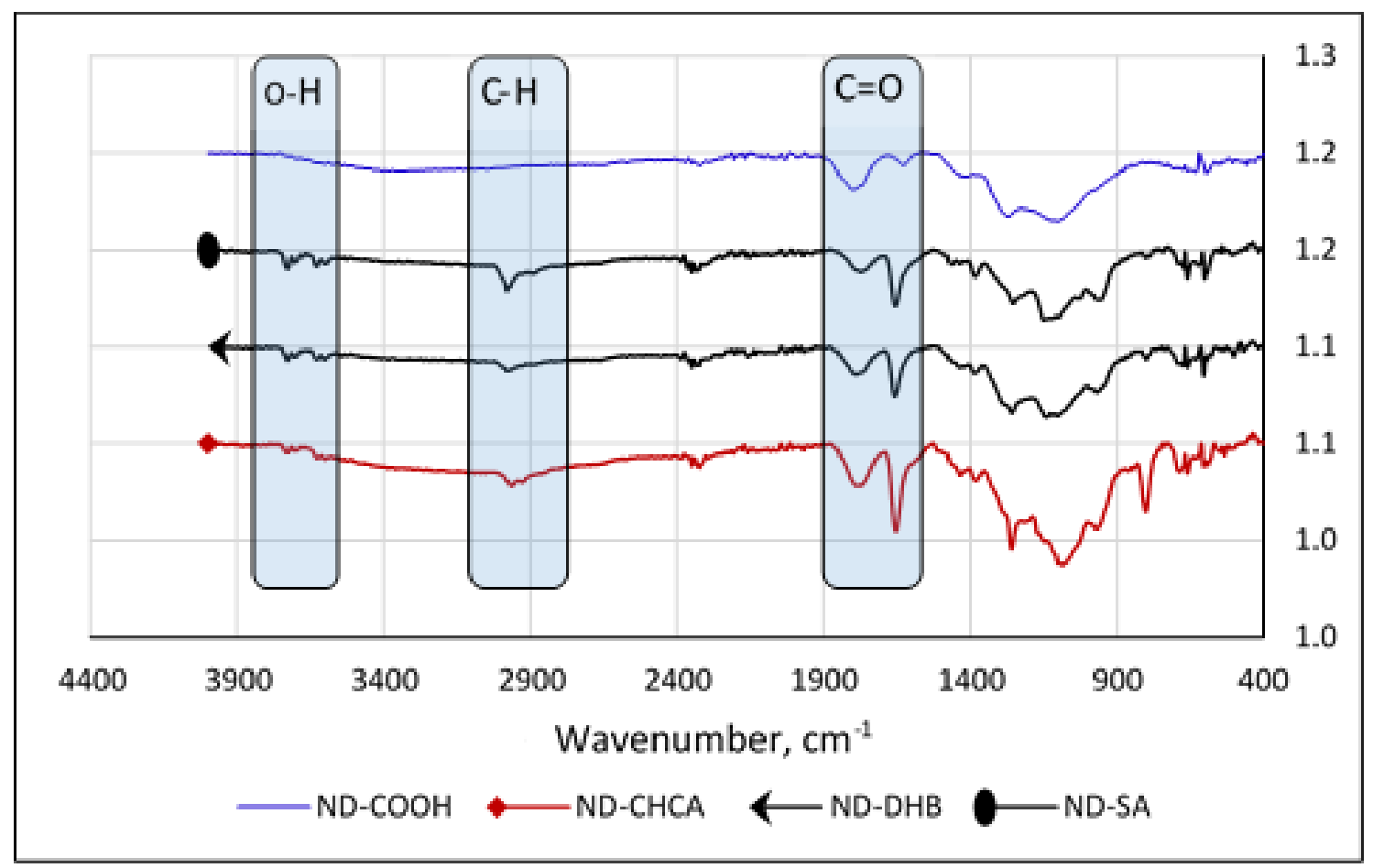

Figure 2: FT-IR spectra for the pristine and functionalized nanodiamonds 
Cite this article as: Chitanda, J.M., Zhang, H., Pahl, E. et al. J. Am. Soc. Mass Spectrom. (2016) 27: 1686.

Final publication is available at Springer via http://dx.doi.org/10.1007/s13361-016-1454-5.

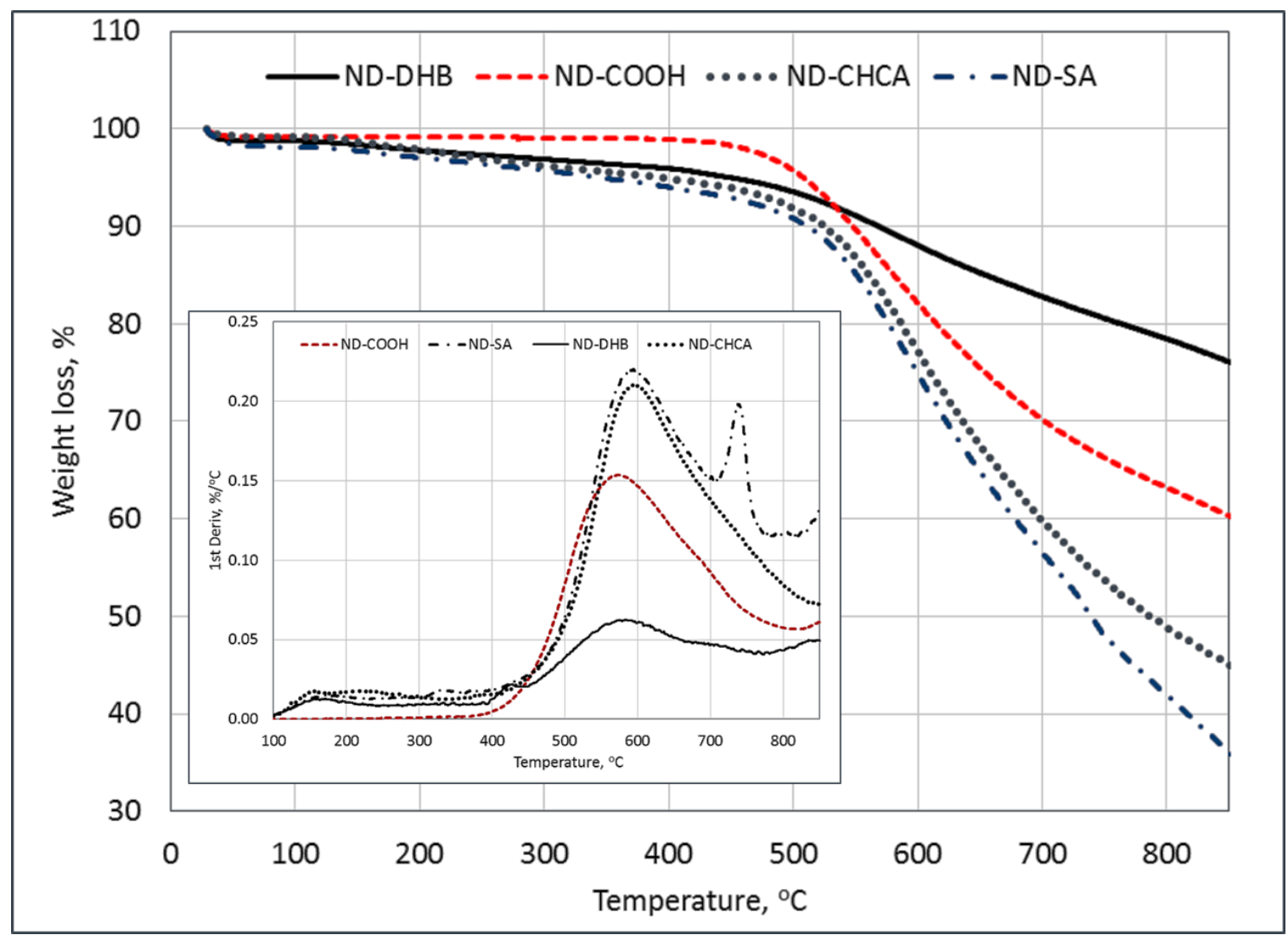

Figure 3: TGA and DTG (insert) graphs for the pristine and functionalized nanodiamonds 
Cite this article as : Chitanda, J.M., Zhang, H., Pahl, E. et al. J. Am. Soc. Mass Spectrom. (2016) 27: 1686. Final publication is available at Springer via http://dx.doi.org/10.1007/s13361-016-1454-5.

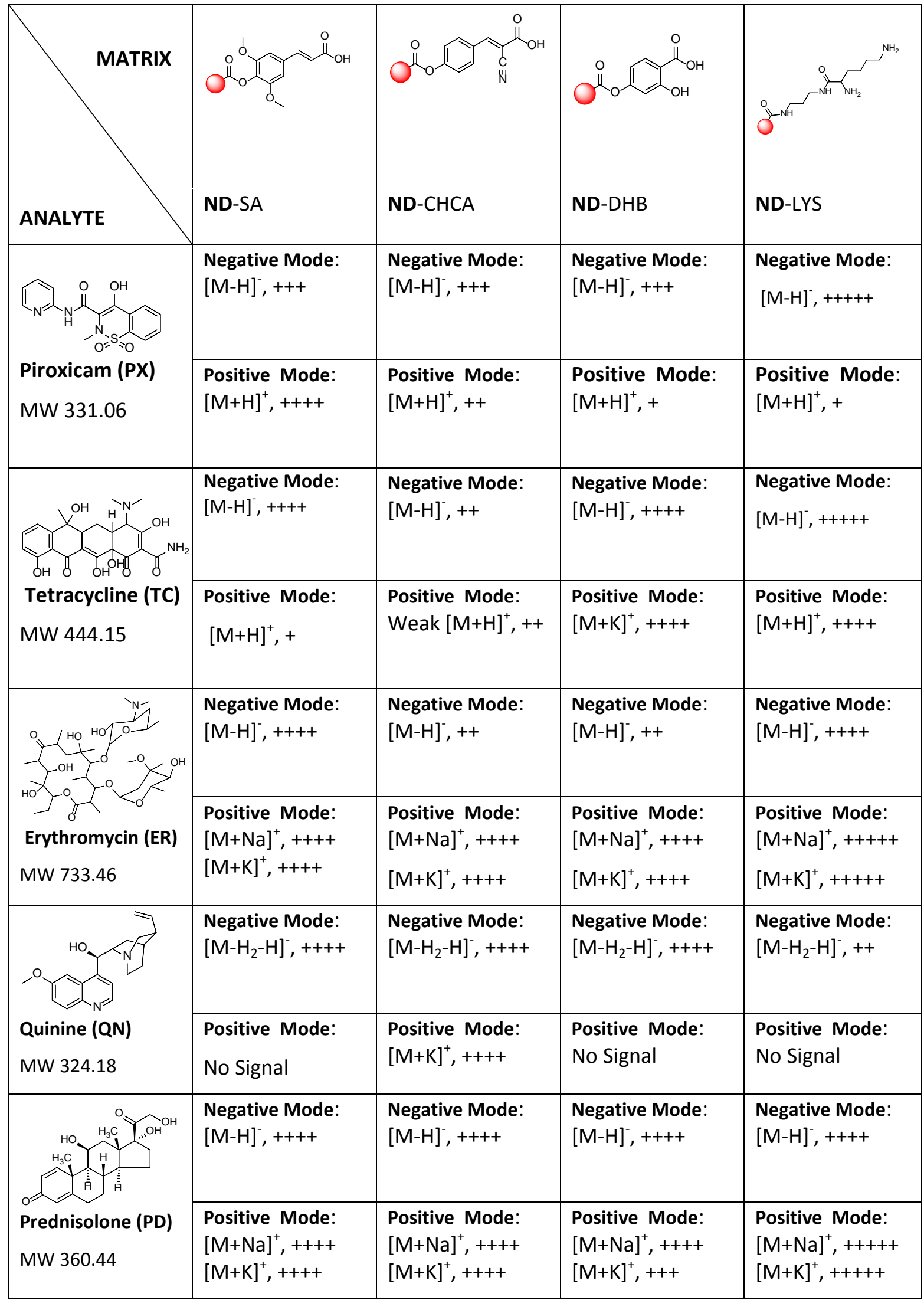

University of Saskatchewan Author Manuscript, available October 1, 2017. 
Cite this article as : Chitanda, J.M., Zhang, H., Pahl, E. et al. J. Am. Soc. Mass Spectrom. (2016) 27: 1686. Final publication is available at Springer via http://dx.doi.org/10.1007/s13361-016-1454-5.

Table 1: Analysis of five analytes using the four nanodiamond-based matrices at laser power of 5300 Laser. Ion intensity was defined based on ion counts as follows: Very intense $\geq 10^{5}$ $(+++++)$, intense $\geq 10^{4}(++++)$, moderate $\geq 10^{3}(+++)$, weak $\geq 10^{2}(++)$, very weak $\leq 10^{2}(+)$. The ball represents the nanodiamond core

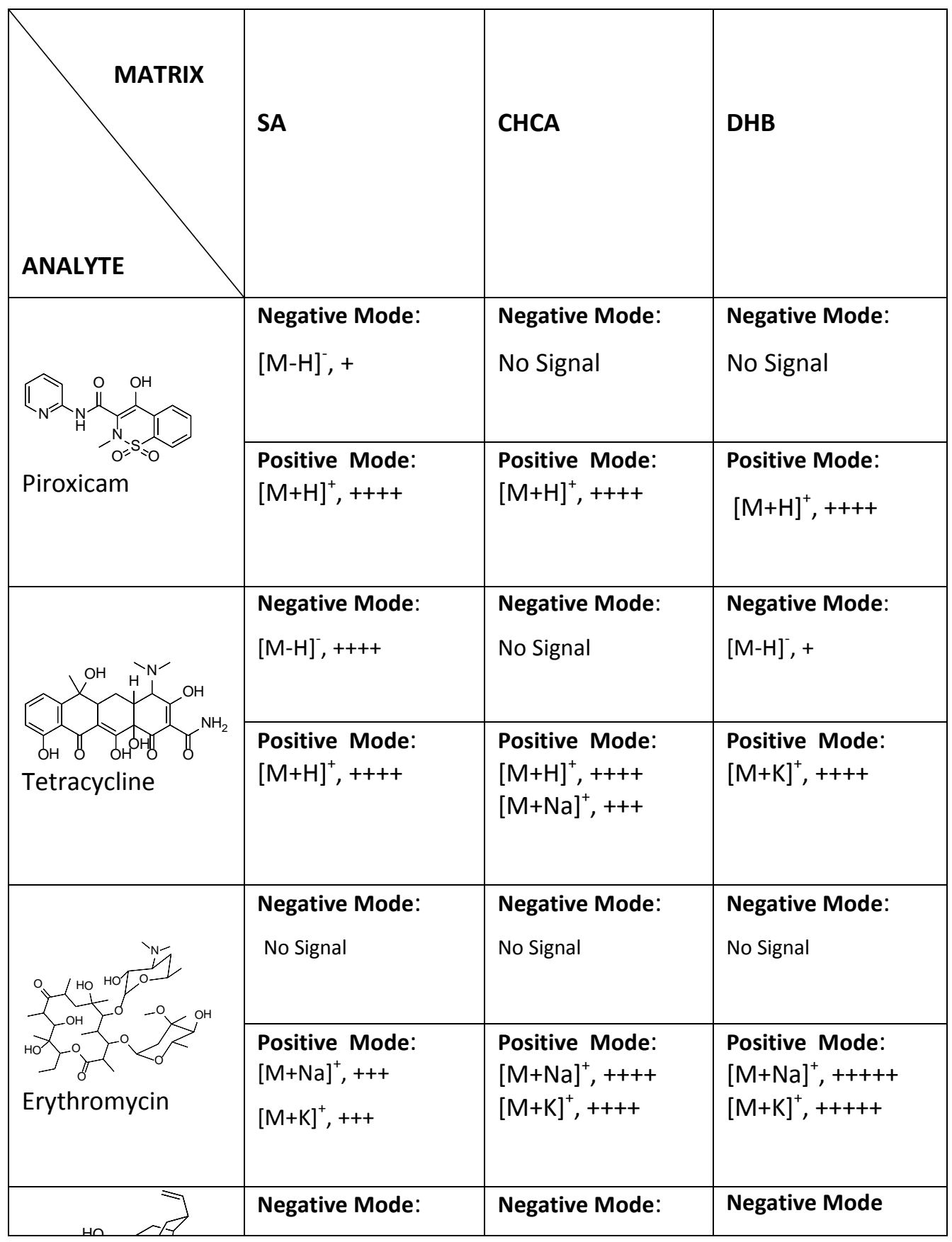


Cite this article as: Chitanda, J.M., Zhang, H., Pahl, E. et al. J. Am. Soc. Mass Spectrom. (2016) 27: 1686. Final publication is available at Springer via http://dx.doi.org/10.1007/s13361-016-1454-5.

\begin{tabular}{|c|c|c|c|}
\hline \multirow[t]{2}{*}{ Quinine } & {$[\mathrm{M}-\mathrm{H}]^{-},++++$} & No Signal & {$[\mathrm{M}-\mathrm{H}]^{-},++++$} \\
\hline & $\begin{array}{l}\text { Positive Mode: } \\
\text { No signal }\end{array}$ & $\begin{array}{l}\text { Positive Mode: } \\
\text { No signal }\end{array}$ & $\begin{array}{l}\text { Positive Mode: } \\
{[\mathrm{M}+\mathrm{Na}]^{+},++}\end{array}$ \\
\hline \multirow[b]{2}{*}{ Prednisolone } & $\begin{array}{l}\text { Negative Mode: } \\
{[\mathrm{M}-\mathrm{H}]^{-},++++}\end{array}$ & $\begin{array}{l}\text { Negative Mode: } \\
{[\mathrm{M}-\mathrm{H}]^{-},++++}\end{array}$ & $\begin{array}{l}\text { Negative Mode: } \\
{[\mathrm{M}-\mathrm{H}]^{-},+}\end{array}$ \\
\hline & $\begin{array}{l}\text { Positive Mode: } \\
{[\mathrm{M}+\mathrm{H}]^{+},++++} \\
{[\mathrm{M}+\mathrm{Na}]^{+},+++}\end{array}$ & $\begin{array}{l}\text { Positive Mode: } \\
{[\mathrm{M}+\mathrm{Na}]^{+},++++} \\
{[\mathrm{M}+\mathrm{K}]^{+},++++}\end{array}$ & $\begin{array}{l}\text { Positive Mode: } \\
{[\mathrm{M}+\mathrm{H}]^{+},++++} \\
{[\mathrm{M}+\mathrm{Na}]^{+},++++} \\
{[\mathrm{M}+\mathrm{K}]^{+},+++}\end{array}$ \\
\hline
\end{tabular}

Table 2: Analysis of five analytes using three common conventional matrices at regular laser power. Ion intensity was defined based on ion counts as follows: Very intense $\geq 10^{5}(+++++)$, intense $\geq 10^{4}(++++)$, moderate $\geq 10^{3}(+++)$, weak $\geq 10^{2}(++)$, very weak $\leq 10^{2}(+)$. 
Cite this article as : Chitanda, J.M., Zhang, H., Pahl, E. et al. J. Am. Soc. Mass Spectrom. (2016) 27: 1686. Final publication is available at Springer via http://dx.doi.org/10.1007/s13361-016-1454-5.

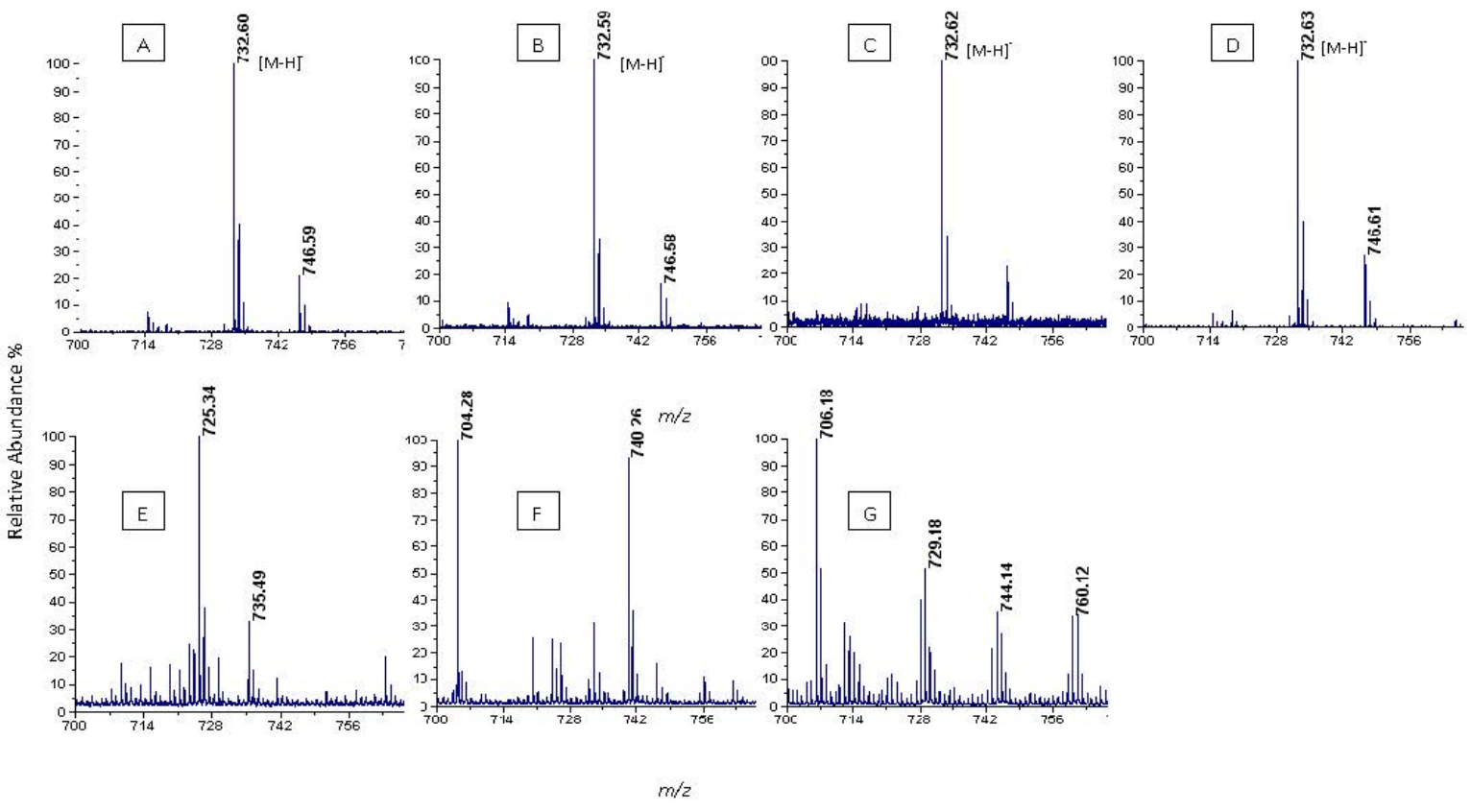

Figure 4: Represent ative mass spectra obt ained in the negative mode for erythrom ycin. The $[\mathrm{M}-\mathrm{H}]]^{-}$peak at $\mathrm{m} / \mathrm{z} 732$ is observed when using nanodiam ond-m odified matrices A) ND-SA; B) ND-CHCA; C) ND-DHB; and D) ND-Lys. Conversely, erythromycin is not observed using conventional matrices E) SA; F) CHCA; and G) DHB 
Cite this article as : Chitanda, J.M., Zhang, H., Pahl, E. et al. J. Am. Soc. Mass Spectrom. (2016) 27: 1686. Final publication is available at Springer via http://dx.doi.org/10.1007/s13361-016-1454-5.

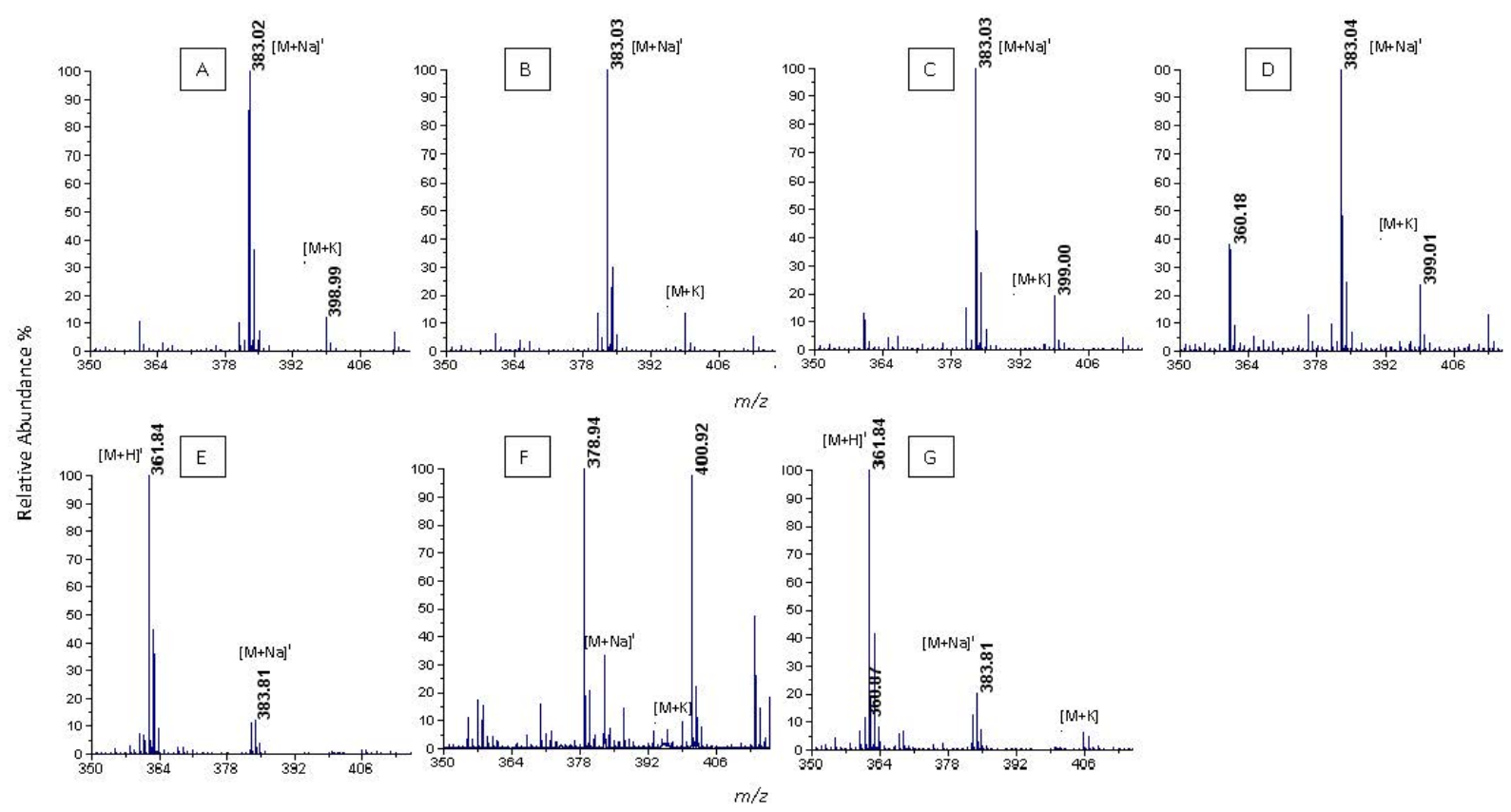

Figure 5: A representative exam ple for the ionization in the positive ion mode for prednisone illustrating the form ation of adducts $[\mathrm{M}+\mathrm{Na}]^{+}$at $\mathrm{m} / 2383$ and $[\mathrm{M}+\mathrm{K}]^{+}$at $\mathrm{m} / 2399$ when using nanodiamond matrices A) ND-SA; B) ND-CHCA; C) ND-DHB; D) ND-LYS. For conventional matrices E) $\mathrm{SA}$; F) CHCA; and $\mathrm{G}) \mathrm{DHB}$, the protonated peak $[\mathrm{M}+\mathrm{H}]^{+}$at $\mathrm{m} / \mathrm{z} 361$ was observed in $\mathrm{E}$ ) and $\mathrm{G}$ ) 